\title{
Differential marking of excision planes in screened breast lesions by organically coloured gelatins
}

\author{
J S Armstrong, I P Weinzwieg, J D Davies
}

\begin{abstract}
Traditionally India ink has been used to mark surgical resection margins, more recently with acetone to aid drying. Alternative methods have been suggested in recent months, including solutions of alcian blue, and Tippex. These methods use one colour only and Tippex is radiodense. Artists' pigments have been used, but their radiodensity makes them unsuitable for the detailed mammograms of the sliced screened specimens. An alternative method was specifically designed for impalpable breast lesions. It is based on coloured gelatins that are painted on to the biopsy specimen before dissection. The markers are radiolucent, quick to set, and do not penetrate the connective tissue. They can be applied to both fresh and fixed tissues, and the cost is about $f 0.05$ a specimen. The system is also suitable for marking other large specimens.
\end{abstract}

To be sure of excising mammographically detected breast lesions the surgeon often resects a considerable volume of breast tissue. Malignant lesions, invasive or in situ, may extend beyond the macroscopic limits and approach or cross the boundaries of surgical excision. It may therefore be necessary to identify accurately the planes of surgical excision. Effective later surgery or the implantation of radioactive substances may therefore depend on the pathological orientation of such lesions. $^{1-3}$

The orientation of the specimen by the surgeon before receipt in the laboratory is usually achieved by inserting either clips or sutures. $^{2}$ A set protocol must be agreed locally. Differing numbers, ${ }^{3}$ or colours of sutures, or differing numbers of knots per suture can be used on three margins of the biopsy specimen to give an accurate three dimensional orientation. The surgeon's practice of using ink to mark the specimen's edge should be discouraged as it obscures the pathologist's own marking.

A technique of differentially marking breast biopsy specimens with inorganic artists' pigments has already been described. ${ }^{4}$ This is very suitable for marking palpable lesions, but the pigments contain heavy metals which are radiodense. The pigment granules are of similar size and density to microcalcification, and may thus be confusing on subsequent specimen mammography after tissue slicing (fig 1).

We were unable to find an alternative method that had been reported, and therefore devised a simple marking system based on gelatins marked by organic pigments that are not radiodense.

\section{Methods}

Agar and gelatin were both initially regarded as possible vehicles for coloured dyes. These were to be painted on to the surface of a biopsy specimen to mark the excision margin. As gelatin has a lower melting point $\left(38^{\circ} \mathrm{C}\right)$ than agar $\left(80^{\circ} \mathrm{C}\right)$ we decided to try gelatin as a coating agent in initial trials. This meant that the marking solution could be melted in warm water, obviating the need for an external heat source.

A $24 \%(w / v)$ solution of gelatin (Porcine gelatin powder, BDH chemicals, Poole, Dorset) was prepared in a 25 millilitre container and $2 \mathrm{~g}$ of the "dye" to be tested was added $(8 \%$ solution $)$. The container was then placed in an oven at $80^{\circ} \mathrm{C}$ for 30 minutes to allow the gelatin to dissolve.

To test the possible leaching of many dyes a small pellet of gelatin was made by allowing $1 \mathrm{ml}$ of the solution to cool and moulding it with the hands. Each pellet was placed in a beaker of formol-saline for 24 hours and then into ethanol for another 24 hours to test for colour leaching in these agents. If the test gelatin solution was considered colourfast a dummy processing was attempted.

The test gelatin and its dye were heated in a beaker of water from the hot tap, for five minutes or until they liquefied. The solution was then painted on to breast tissue using a squirrel hair artists' watercolour brush (size 5). The gelatin was applied in several coats to a depth of about $1 \mathrm{~mm}$. There was no need to allow time for drying between coats. The gelatin was allowed to set and the coated specimens were placed in formol-saline for 24 hours. Blocks were then taken from the tissue to include the painted margins and placed in standard sized $(25 \times 30 \times 5 \mathrm{~mm})$ plastic cassettes (Ames, Slough, England). In the initial trials these blocks were then individually hand processed using graduated ethanols and toluene, sectioned, rehydrated and then cleared using ethanol and xylene. ${ }^{5}$ The processing fluids were all examined for colour contamination. Finally sections were cut and stained with Harris's haematoxylin and eosin. 

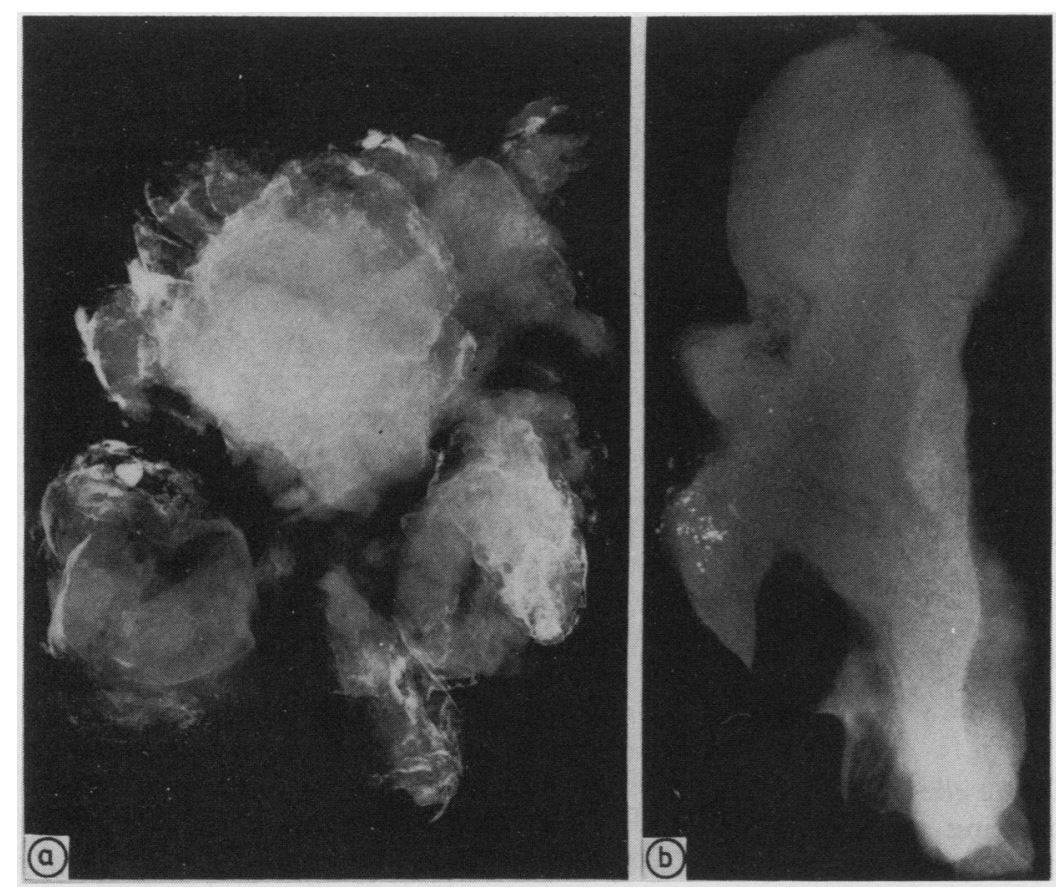

Figure 1

Photoradiograph of $3 \mathrm{~mm}$ slices of breast tissue painted with inorganic artists' pigments in acetone (a). The opacities carried across the tissue slice may mimic or obscure the microcalcification seen in intraduct carcinoma $(b)$

The colours were then assessed both macroscopically and microscopically.

All the chosen gelatins regarded as suitable marking agents were then used to mark breast tissue differentially and were processed among routine material as described above.

The colours were then reassessed by the authors, particularly for ease of discrimination between colours after staining. Finally the differential marking system evolved was used prospectively on a consecutive series of 50 breast biopsy specimens. The specimens were reported by the reporting team on duty and subsequently examined by the authors.

\section{Results}

Agar was inconvenient to use as a vehicle due to its high melting point and no further work was attempted with it. All the solutions used were radiolucent.

In the initial solubility testing experiment many dyes were excluded due to their solubility in $10 \%$ formol saline or alcohol (Appendix). Eight reagents were considered suitable for testing on tissue (table 1). Those eventually selected were Bismark brown, Orange G, Janus green, and powdered commercial paprika, turmeric and henna; uncoloured gelatin and a suspension of India Ink in gelatin were also used.

Table 1 Properties of eight suitably stable gelatins when painted on to breast tissue and processed and stained in a routine manner

\begin{tabular}{|c|c|c|c|}
\hline \multirow[b]{2}{*}{ Compound } & \multicolumn{2}{|c|}{ Colour and appearance } & \multirow{2}{*}{$\begin{array}{l}\text { Stability (during } \\
\text { fixation and processing) }\end{array}$} \\
\hline & Macroscopic & Microscopic & \\
\hline Bismark brown & Brown & Brown particles & $\begin{array}{l}\text { Some staining of the } \\
\text { formalin }\end{array}$ \\
\hline Janus green & Blue & Purple particles & Good \\
\hline Indian ink & Black & Black particles & Good \\
\hline Orange $G$ & Yellow & Diffuse brown & Good \\
\hline Gelatine & Colourless & Diffuse pink/purple & Good \\
\hline Paprika & Red/brown & $\begin{array}{l}\text { Red pigmented } \\
\text { cellulose fragments }\end{array}$ & Good \\
\hline $\begin{array}{l}\text { Turmeric } \\
\text { Henna }\end{array}$ & $\begin{array}{l}\text { Yellow } \\
\text { Brown }\end{array}$ & $\begin{array}{l}\text { Cerebriform starch granules } \\
\text { Brown pigmented } \\
\text { cellulose fragments }\end{array}$ & $\begin{array}{l}\text { Good } \\
\text { Good }\end{array}$ \\
\hline
\end{tabular}

The colour visible macroscopically was often modified by staining with eosin when examined microscopically. This made discrimination between some dyes, such as Orange $\mathrm{G}$ and Bismark brown impossible. In general, it was easier to discriminate between the colours of particulate dyes: This explains the success of the plant materials used. After a short period all trainee and senior members of the department could identify the individual solutions histologically.

There was some leaching of colour into the connective tissue planes, but the edges were clearly indicated by the solid gelatin line. Leaching into the tissue was more severe if a weaker gelatin solution was used and with some of the more soluble dyes, such as Bismark brown, (table 1 ).

If the brush was inadequately cleaned between each solution colour carry-over occurred, making microscopic discrimination difficult or impossible.

To mark an impalpable lesion differentially six colours need to be used. One for the medial, lateral, superior and inferior margins, and one each for the superficial and deep margins. In practice the six best gelatins were those with India ink, Janus green, Bismark brown, turmeric, henna, and plain gelatin. Using these six coloured solutions it was possible to mark the resection margins so that they were clearly differentiated macroscopically (fig 2), and microscopically in the processed block (fig 3).

Bismark brown was characterised by particles of brown pigment, Janus green by particles of blue to violet pigment, and India ink by black particles. Plain gelatin stains both with haematoxylin and eosin, hence its colour is a homogenous pink to faint purple. The plant substances were identified by their morphological characteristics. Henna is a powder derived from the dried leaves of the Egyptian privet Lawsonia ineris. A brown pigment is seen within the cellulose fragments. Turmeric is an extract derived from the rhizome of the plant Curcuma longa and contains characteristic cerebriform starch granules. Paprika consists of ground seed cases. It is a heterogeneous substance; the form used here is the commonly available mild Hungarian type from Capcicum annuum. The plant particles contain a vivid red pigment. A number of distinct colours and particulate patterns are seen microscopically within the gelatin matrix.

Painting the biopsy specimens was quick and easy and the paint brushes were easily washed in warm water. The gelatin suspensions or dyes were easy and clean to use, there was no staining of the implements used, and the solutions all washed off in warm water.

The marking caused few problems in cutting or staining of the formalin fixed tissue. The turmeric particles caused minimal scratching of the microtome blade. When fresh tissue was marked and subsequently sampled for frozen section examination, the gelatin stuck to the scalpel. Pre-cooling the biopsy specimen solved this problem. The gelatin on the surface, however, made cutting a high quality frozen section technically demanding. If the gelatin 


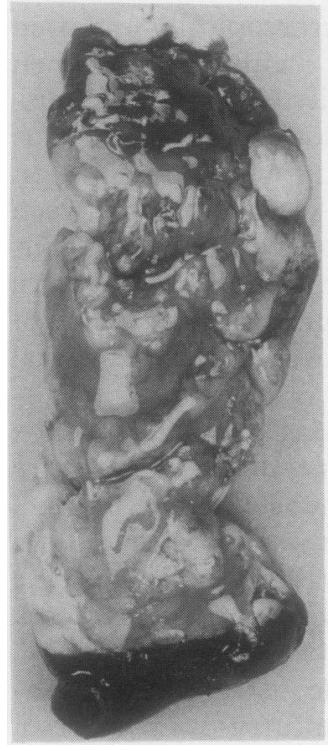

Figure 2 A breast biopsy specimen, $10 \mathrm{~cm}$ long, differentially marked with Bismark brown (superficial), India ink (deep), plain gelatin (medial), henna (lateral and paprika (superior). The set gelatin is responsible for the glistening appearance.
Table 2 Drying or setting times of various marking substances

\begin{tabular}{ll}
\hline Substance & Time to dry \\
\hline Gelatin-tissue at room temperature & $<5$ minutes \\
Gelatin-chilled tissue & $<10$ seconds \\
Pigments in acetone & 20 minutes \\
Indian ink & 30 minutes \\
Silver nitrate & Blots dry \\
\hline
\end{tabular}

was peeled off the block chosen for frozen sectioning this problem was avoided. No difficulty was experienced in sectioning of the frozen tissue when subsequently fixed for the preparation of permanent sections.

The gelatin setting time was assessed and compared very favourably with artists' pigments suspended in acetone, ${ }^{4}$ particularly when the biopsy specimen was chilled (table 2).

\section{Discussion}

Our aim was to find a simple effective method for the differential marking of mammographically detected breast biopsy specimens. Ideally, it should also be cheap and non-toxic.

Any substance to be used for marking has to fulfil several basic criteria. It has to be relatively insoluble in $10 \%$ formol saline, ethanol, toluene, paraffin wax and xylene to survive processing and to prevent unacceptable contamination of the reagents. It has to remain on the surface of the biopsy specimen and be clearly visible both macroscopically and microscopically. Most importantly for our purpose it has to be radiolucent.

To be radiolucent a substance must have a relative molecular mass (RMM) less than that of the "window" of the $x$-ray machine. In mammography the element used in the machine as a "window" is beryllium (RMM of 4). ${ }^{6}$ Calcium has an RMM of 20 and is seen

Figure 3

Photomicrograph of a breast biopsy specimen with the resection margin marked with a solution of gelatin coloured with turmeric. Note the distinctive cerebriform starch particles.

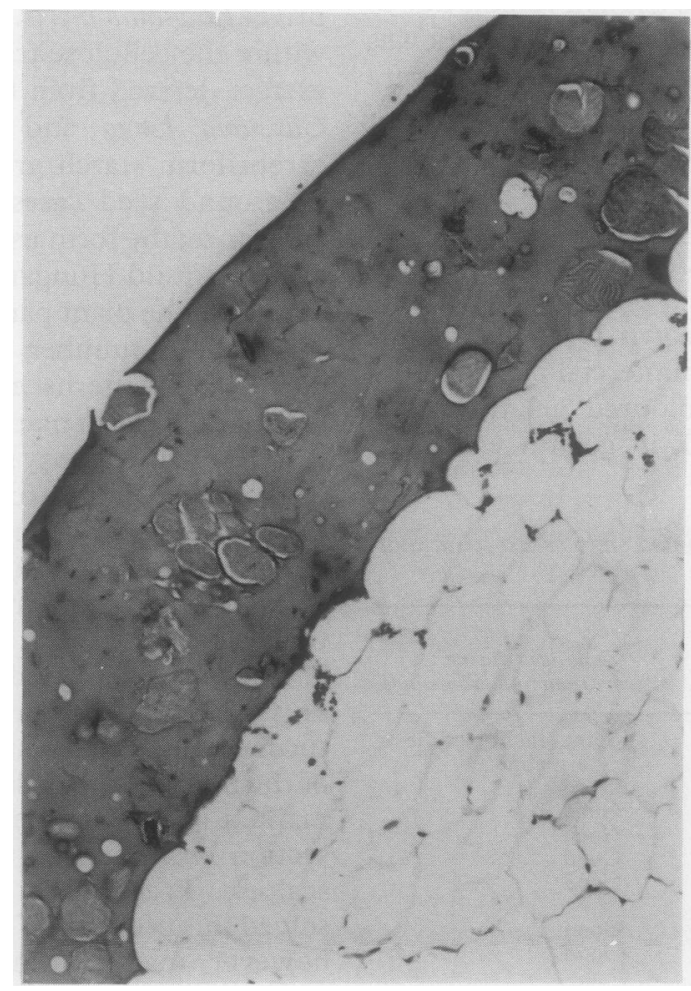

clearly on mammography. For practical purposes carbon with an RMM of 14 is invisible. We were unable to find sufficient inorganic pigments composed of elements of suitable character in this lower range to use for differential marking. As an alternative to inorganic pigments, many synthetic organic dyes were tested but these were soluble in formol saline (Appendix).

In staining small biopsy specimens mounted in $\operatorname{agar}^{7}$ for mucin histochemistry it was noticed that certain dyes were retained preferentially in the agar. We adopted the basis of this method by dissolving dyes in a gel. The gel dyes are rendered insoluble because they bind to the protein in the solution. We used this property to keep the dyes on the surface of the biopsy specimen and thus prevent leaching of dye into the processing solutions or tissue. Initially collagen type dyes were used. In an attempt to obtain more colours other substances were subsequently tried on a purely empirical basis.

The gelatin set in a few minutes at room temperature and prechilling the biopsy specimens resulted in almost instantaneous setting of the solutions or suspensions. Subsequent depositing of the biopsy specimens in formol saline resulted in polymerisation of the gelatin and improved the results. The method was effective for both fresh and fixed tissue.

All members of the department have found the identification of the margins of surgical excision both straightforward and accurate. In fact, the method was preferred to other marking systems such as India ink, silver nitrate, and artists' pigments suspended in acetone. ${ }^{4}$

The demarcation between two adjacent colours was obvious both macroscopically and microscopically. Mistakes in painting can also be rectified by painting a second colour on top of the first.

Differentiation between some colours was easier than others. Janus green, India ink, and plain gelatin were easily identified. There were initial difficulties in differentiating Orange $G$ and Bismark brown from the plain gelatin. The particulate nature of the Bismark brown made the identification easier. Only modest knowledge of botany was required for the identification of the three plant colouring materials.

Obviously a set local protocol needs to be devised and used consistently. In many cases full differential marking is not indicated, but some differentiation may be appropriate. Colouring a spare piece of tissue with the gelatins to be used will also allow the pathologist to become familiar with the colours.

It is important to wash the paintbrush thoroughly between each solution to avoid colour contamination; furthermore, it is simpler to have a separate brush for each solution.

A $25 \mathrm{ml}$ container of gelatin and dye costs $£ 0.35$-that is, about $£ 0.05 \mathrm{p}$ a specimen. The time taken to mix the solution by the laboratory staff was only a few minutes. By adding a few grains of thymol to the solutions they can be kept almost indefinitely, but addition of thymol to the Janus green solution rendered it soluble in formalin and for this reason its use was 
abandoned. The solutions are kept in a lidded plastic container and stored at room temperature in the dissection room. When needed they are placed in a beaker of warm water from the hot tap for a few minutes to melt. A $25 \mathrm{ml}$ container of each marker will paint up to 30 biopsy specimens.

Some of the synthetic dyes used are toxic, but once in the gelatin base aerosol formation is not a problem. Gloves should be used when handling the solutions.

This is a simple and cheap method of marking the resection margins of breast specimens. Furthermore, it eliminates the potential hazard of toxic heavy metal inorganic pigments. This method could be successfully applied to other large specimens either as a single colour or as a differential marking system. Plain gelatin can be used alone for biopsy specimens that do not require differential marking.

The marking system permits detailed correlation with mammograms, and because of the lack of penetration into connective tissue planes, effective adhesion, and rapid setting, the method can be applied to fresh tissue.

\section{Appendix}

Compounds found unsuitable for staining gelatin.

Leached in formol saline

Acid fuchsin (CI 42685), Aniline blue WS (CI 42755), Basic fuchsin (CI 42500), Carbol fuchsin, Malachite green (CI 42000), Martius yellow (CI 10315), Methylene blue (CI 52015), Phloxine (CI 45405), Ponceau S (CI 27195), Safranin, Sirius red 4B (CI 28160), Solochrome Cyanine $\mathbf{R}$ (CI 43820), Tartrazine (CI 19140), Toluidine blue (CI 52040).

Leached in ethanol

Sudan black B (CI 26150), Sudan III (CI 26100).

Coagulated the gelatin

Alcian blue (CI 74240).

Leached into the tissue planes

Carmine (CI 75470).

1 Sloane JF, et al. NHS breast screening programme: guidelines for pathologists. Oxford: NHS/BSP. 1990.

2 Davies JD. Pathology and screened breast lesions. Curr Pract Surg 1989;1:147-51.

3 Bogomoletz WV, Davies JD. L'etude macroscopique des pieces de resection mammaire: une approche moderne. Ann Pathol (Paris) 1990;10:(in press).

4 Paterson DA, Davies JD. Marking planes of surgical excision on breast biopsy specimens: use of artists' pigments suspended in acetone. J Clin Pathol 1988;41: 1013-16.

5 Davies JD, Young EW, Mera SL, Barnard K. Lysozyme is a component of vascular elastic fibres. Experientia 1983;39:382-3.

6 Rosen PP, Snyder RE, Robbens G. Specimen radiology for nonpalpable breast lesions found by mammography: procedures and results. Cancer 1974;34:2028-33.

7 Vasisht HS, Dawar BL. An agar matrix for precise orientation and subsequent paraffin embedding of unicellular tion and subsequent parafin embeddin. 